1 Failure of VITEK2 to reliably detect vanB-mediated vancomycin resistance in

\title{
Enterococcus faecium
}

3

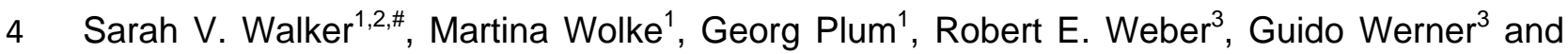

5 Axel Hamprecht ${ }^{1,2,4}$

$6{ }^{1}$ Institute for Medical Microbiology, Immunology and Hygiene, University Hospital of Cologne,

7 Cologne, Germany

$8 \quad{ }^{2}$ DZIF (German Centre for Infection Research), partner site Bonn-Cologne, Germany

$9 \quad{ }^{3}$ Department of Infectious Diseases, Division of Nosocomial Pathogens and Antibiotic

${ }^{4}$ Institute for medical microbiology and virology, University of Oldenburg, Oldenburg,

Germany

${ }^{\#}$ Corresponding author:

Dr. Sarah Victoria Walker

Institute for Medical Microbiology, Immunology and Hygiene, University Hospital of Cologne

current contact information:

\section{Running title}

Low-level vancomycin resistant VRE unreliably detected by Vitek2

Keywords: 


\section{Abstract}

Objectives: The increasing prevalence of vancomycin resistant enterococci (VRE) necessitates a reliable detection of VRE especially for low level resistance mediated by vanB in Enterococcus faecium. In this prospective study we analyzed if $\operatorname{van} B$ mediated vancomycin resistance can be reliably detected by Vitek2.

Methods: 1344 enterococcal isolates from routine clinical specimens were tested by Vitek2 (bioMérieux, Nürtingen, Germany). Additionally, a bacterial suspension ( 0.5 McFarland) was inoculated on a chromID VRE screening agar (bioMérieux) and incubated for 48 hours. If vancomycin was tested susceptible by Vitek2 but growth was detected on the screening agar a PCR for vanA/vanB was performed (GeneXpert vanA/B test kit, Cepheid, Frankfurt, Germany). MICs of vancomycin susceptible by Vitek but van $A / B$ positive isolates were determined before and after cultivation in a broth with increasing concentration of vancomycin.

Results: $156 / 492$ of E. faecium were VRE, predominantly vanB (87.0\%) of which 14 were not identified as VRE by Vitek2 (sensitivity $91.0 \%$ ). The majority (9/14) demonstrated high-level MICs by broth dilution. Even after exposure to increasing vancomycin concentrations MICs remained nearly identical. Three of the undetected isolates demonstrated initial growth on chromID VRE, after the vancomycin exposure additional 7 isolates demonstrated growth on chromID VRE.

Conclusions: Vitek2 fails to detect vanB mediated vancomycin resistance consistently, especially but not limited to low-level resistance. As this may lead to treatment failure and further dissemination of vanB VRE, additional methods (e.g. culture on VRE screening agar or PCR) are necessary to reliably identify vanB-positive enterococci in clinical routine. 


\section{Introduction}

Over the last decade vancomycin resistant Enterococcus faecium (VREfm) have become increasingly prevalent causing infections especially in immunocompromised patients. ${ }^{1,2}$ The endemic occurrence of VREfm and increasing number of outbreaks with only limited remaining therapeutical options led to the WHO classification as a high priority pathogen in 2017..$^{3,4}$

The reliable detection of VRE is essential for the initiation of adequate therapy and infection control measures. However, especially low level resistance mediated by van $B$ in Enterococcus faecium is difficult to detect (hereafter referred to as "occult VRE") as the minimal inhibition concentration (MIC) does not necessarily resemble phenotypic vancomycin resistance. ${ }^{5}$ In July 2018 EUCAST issued a warning against the use of vancomycin gradient tests to detect vancomycin resistance in E. faecium and E. faecalis. ${ }^{6}$

In this prospective study we analyzed if vanB mediated vancomycin resistance can be reliably detected by Vitek2 and whether vancomycin resistance can emerge after vancomycin exposure in vitro. This is essential for patient management as vancomycin remains the first line antibiotic for treatment of $E$. faecium infections. ${ }^{7}$

\section{Materials/methods}

From 10/2018 until 05/2019 (8 months) 1344 non-copy enterococcal isolates from routine clinical specimens were tested prospectively using the AST-P592 card in a Vitek2 system (bioMérieux, Nürtingen, Germany). Additionally, a bacterial suspension equivalent to 0.5 McFarland standard was inoculated on a chromID VRE screening agar (bioMérieux) and incubated for 48 hours. If vancomycin susceptibility was determined by Vitek2 (MIC $\leq 4 \mathrm{mg} / \mathrm{L}$ ) but growth was detected on the VRE screening agar a PCR for vanA/vanB was performed using the GeneXpert vanA/B test (Cepheid, Frankfurt, Germany). In case of vanA or vanB detection, isolates were classified as VRE. For isolates which were positive for vanA or vanB but phenotypically vancomycin susceptible the vancomycin MIC was further assessed by broth microdilution using the MICRONAUT-S MRSA/GP plate (Merlin Diagnostika, Bornheim, 
Germany) and vancomycin MIC test strips according to the macromethod (Liofilchem, Roseto degli Abruzzi, Italy) as recommended by the manufacturer and previously described. ${ }^{8}$ For occult VRE, results of susceptibility testing and VRE PCR were also verified at the National Reference Centre for Enterococci at the Robert Koch Institute.

Additionally, to analyze if any VRE were missed using regular susceptibility testing or by VRE screening agar and to ensure methodical consistency, all available E. faecium (243/491) from routine clinical specimens isolated between March to May 2019 were analyzed for vanA/vanB presence using the Anyplex VanR real-time Detection kit (Seegene, Düsseldorf, Germany), irrespective of whether growth on the VRE screening media was observed.

Furthermore, we compared if follow-up isolates of patients with occult VRE developed phenotypic vancomycin resistance.

\section{Induction of vancomycin resistance}

Phenotypically vancomycin susceptible but van $A / B$ positive isolates were grown in brainheart-infusion broth supplemented with vancomycin in increasing concentrations $(1 \mathrm{mg} / \mathrm{L}, 2$ $\mathrm{mg} / \mathrm{L}, 4 \mathrm{mg} / \mathrm{l}, 10 \mathrm{mg} / \mathrm{l}$ and $25 \mathrm{mg} / \mathrm{L})$ for $48 \mathrm{~h}$ at $36^{\circ} \mathrm{C}$ in a rotary shaker at $200 \mathrm{rpm}$. If growth was detected, blood agar plates were inoculated with broth cultures containing the highest vancomycin concentration. Subsequently, the vancomycin MIC of vancomycin exposed isolates was determined by microdilution and growth on chromID VRE was tested (supplementary fig. 1).

\section{Results}

The majority of screened isolates $(63.5 \%$; 853/1344) were E. faecalis of which none were VRE (0\%) (Figure 1). Therefore, E. faecalis isolates were not further investigated. In contrast, for E. faecium 156 out of 491 isolates were identified as vancomycin resistant $(31.8 \%)$ (Figure 1).

Among all tested E. faecium isolates, vanB was predominant: $87.0 \%(94 / 108)$ were vanB, $17.6 \%(19 / 108)$ vanA and $2.8 \%(3 / 108)$ possessed both resistance determinants. 
Vitek2 did not detect 14 low-level resistant E. faecium (occult VRE); of these, three were detected by growth on the VRE screening agar (Table 1). The sensitivity of Vitek2 for VRE detection was $91.0 \%(142 / 156)$ whereas the chromID screening agar's detection rate was $93.6 \%(146 / 156 ; p=0.52)$.

As shown in Table 1, nine of the 14 isolates with low-level vancomycin MICs demonstrated high-level MICs by broth microdilution. The remaining five isolates showed low-level vancomycin MICs both in Vitek2 and broth microdilution (Table 1). Although MICs determined by MIC test strips varied from the MICs by Vitek2 and broth microdilution (Table 1), no very major errors were observed (classification as false vancomycin susceptible).

Prior to an additional MIC screening by broth microdilution, 14 occult VRE were exposed to vancomycin in increasing concentrations. Interestingly, the MIC of exposed strains remained nearly identical to the MIC of the original strains when tested by broth microdilution (Table 1). Seven of 11 occult VRE which initially did not grow on the VRE screening media demonstrated growth after vancomycin exposure. These seven isolates were also showed high-level vancomycin MICs in the broth microdilution and grew in the brain-heart-infusion broth with the maximum vancomycin supplement $(25 \mathrm{mg} / \mathrm{L})$.

123 For eight of the 14 patients with initially occult VRE follow up specimens were available. In four out of these eight patients VRE with high-level vancomycin MICs were detected within one month. Unfortunately, clinical data on antimicrobial therapy of the patients (e.g. glycopeptide therapy) was not available.

\section{Discussion}

129 Over the last decades, vancomycin resistant E. faecium have emerged as a new potentially 130 life-threatening pathogen especially in immunocompromised patients. ${ }^{1,2}$ Due to their 131 significance for therapy and infection control, a reliable detection of this pathogen is essential 132 for patient health. In this real-life prospective study, we demonstrate that VREfm frequently remain undetected by Vitek2 and chromID VRE. Additionally, not only low-level resistant 
VREfm remain undetected but also MICs of high-level vancomycin resistant VREfm (HLVRE) were frequently underestimated (9/14 isolates). ${ }^{8}$ All isolates not identified as VRE by Vitek2 were vanB type (14/14).

Interestingly, in isolates possessing van genes but without phenotypic vancomycin resistance, MICs did not increase significantly after exposure to vancomycin. We expected that vancomycin exposure may lead to an enhanced induction of the $v_{a n} H_{B} B X_{B}$ operon, resulting in development of phenotypic resistance against vancomycin, as previously reported for vancomycin-variable E. faecium (VVE) ${ }^{9-12}$ The occult VRE in the present study were unlike VVE which are vanA VREfm reverting from a VSE phenotype to VRE by constitutive expression of the vancomycin resistance cassette..$^{9}$ VVE possess vanA, demonstrate low vancomycin MICs, do not grow on VRE screening media and may cause treatment failure if treated with vancomycin..$^{9-11,13}$ In 2018, Hashimoto et al. described a stealthy vanB cluster which consisted of vancomycin susceptible $E$. faecium (MIC $=3 \mathrm{mg} / \mathrm{L}$ ) that possessed the ability to spontaneously revert to a resistant phenotype mainly caused by a change of $\operatorname{van} B$ gene transcription levels. ${ }^{14}$ The majority of our occult VRE only demonstrated low-level MICs by Vitek2 but high MICs by broth microdilution or MIC test strips indicating an existing high-level vancomycin resistance. Unlike stealthy $\operatorname{VRE}^{14}$ or vancomycin-variable VRE ${ }^{9-11}$ occult VRE were mainly VRE evading detection by Vitek2.

As the MIC determination by Vitek2 is merely a deduction of three antibiotic concentrations in comparison to reference data instead of a single endpoint reading, the uniqueness of occult VRE may not be based on a variation of the van gene but on their growth capacity. Vitek2's inadequacy to detect occult VRE may therefore be based on inapt reference data. As undetected VRE pose a threat for treatment failure for patients with VRE infections possible evasion mechanisms need to be further investigated.

The clinical significance of the van gene detection in the five isolates which were vanB positive but had low vancomycin MICs despite repeated exposure to vancomycin remains unclear. Interestingly, we identified 4 cases who initially had occult VRE and within one 
month developed VRE which were detected by Vitek. Unfortunately, it was not possible to collect and compare these isolates to the original strains.

Until now, to the best of our knowledge, no data is available on the outcome of patients infected by occult VRE who are treated with vancomycin. In an outbreak among neonatal patients in Germany the existence of phenotypic and genotypic discrepancies in vanB positive E. faecium was reported. The outbreak was only detected fortuitously and a colonization rate between $10-25 \%$ was assumed. ${ }^{15}$ In this study only specimen associated with clinical infection were included and a prevalence of almost $10 \%$ was recorded. The colonization with occult VRE is bound to be higher and may be the reason for the extensive spread of vanB positive VRE in Germany beginning in $2007 . .^{4,15}$

In conclusion, our data demonstrates the inability of Vitek2 to reliably detect even high-level vancomycin resistance in $E$. faecium that is associated with the vanB genotype. Additional methods (e.g. culture on VRE screening agar or PCR) are necessary to improve detection of van-positive enterococci in clinical routine and to avoid treatment failure. As vanA/B PCR for all clinical E. faecium isolates is neither feasible nor cost-efficient in the setting of a routine lab, subculture on VRE screening media represents a pragmatic solution and increases VRE detection rates. Therefore, further studies are needed to determine which screening agar performs best for VRE detection in combination with Vitek2. Considering that $9 \%$ of the VRE in this study were occult VRE we recommend performing vanA/B PCR of all E. faecium isolated from invasive infections (including all blood cultures).

\section{Acknowledgements}

We thank all technicians of the diagnostic laboratory of the Institute for Medical Microbiology, Immunology and Hygiene and at the National Reference Centre for excellent technical assistance.

\section{Funding}

This study was done as part of routine diagnostic work and no specific funding was received. 
190 The authors have nothing to declare.

191

Figure 1: Species distribution of enterococcal isolates in routine clinical specimen $(10 / 2018-05 / 2019)$

\section{VRE/VSE distribution of screened isolates}

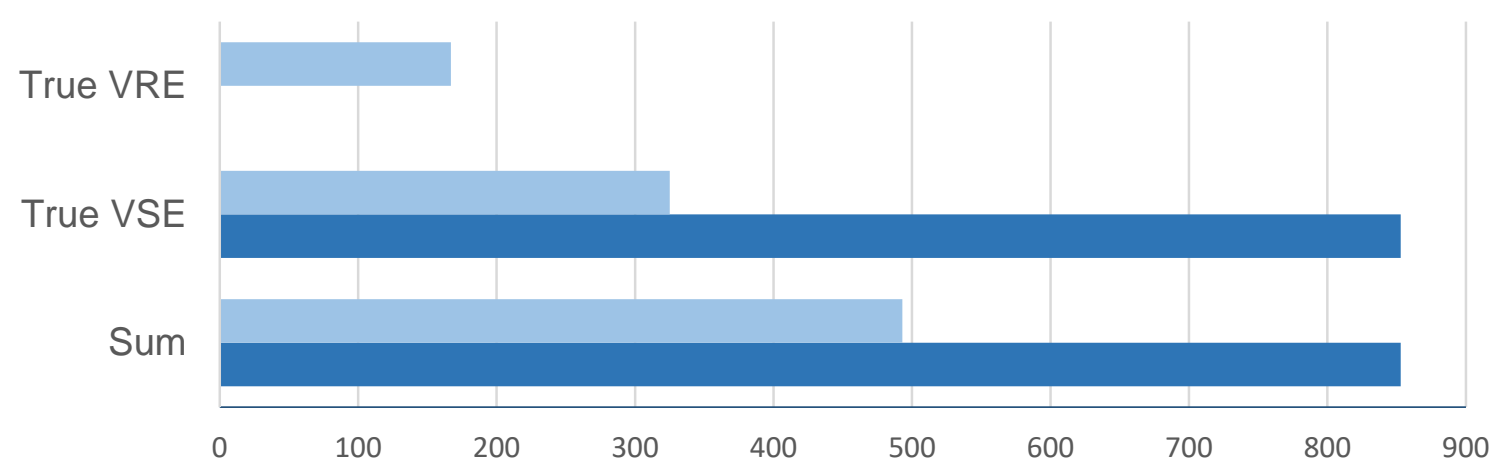

\begin{tabular}{|c|c|c|c|}
\cline { 2 - 4 } \multicolumn{1}{c|}{} & Sum & True VSE & True VRE \\
\hline E. faecium & 493 & 325 & 167 \\
\hline E. faecalis & 853 & 853 & 0 \\
\hline
\end{tabular}

194

195

196

197 


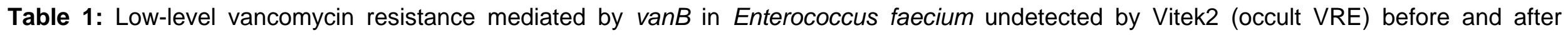
vancomycin exposure.

\begin{tabular}{|c|c|c|c|c|c|c|c|c|c|}
\hline \multirow{3}{*}{ isolate } & \multicolumn{6}{|c|}{ before vancomycin exposure } & \multicolumn{3}{|c|}{ after vancomycin exposure } \\
\hline & \multirow{2}{*}{$\begin{array}{l}\text { Growth } \\
\text { on } \\
\text { chromID } \\
\text { screening } \\
\text { agar } \\
\end{array}$} & \multirow{2}{*}{$\begin{array}{l}\text { vancomycin } \\
\text { MIC by broth } \\
\text { microdilution } \\
{[\mathrm{mg} / \mathrm{L}]}\end{array}$} & \multirow{2}{*}{$\begin{array}{c}\text { vancomycin } \\
\text { MIC by } \\
\text { Vitek2 [mg/L] }\end{array}$} & \multicolumn{2}{|c|}{$\begin{array}{l}\text { vancomycin MIC by MIC test } \\
\text { strip [mg/L] }\end{array}$} & \multirow{2}{*}{$\begin{array}{c}\text { Growth in BHI } \\
\text { supplemented } \\
\text { with vancomycin } \\
{[\mathrm{mg} / \mathrm{L}]}\end{array}$} & \multirow{2}{*}{$\begin{array}{l}\text { Growth on } \\
\text { chromID } \\
\text { screening } \\
\text { agar }\end{array}$} & \multirow{2}{*}{$\begin{array}{l}\text { vancomycin } \\
\text { MIC by broth } \\
\text { microdilution } \\
{[\mathrm{mg} / \mathrm{L}]}\end{array}$} & \multirow[t]{2}{*}{$\begin{array}{l}\text { vancomycin MIC by } \\
\text { Vitek2 [mg/L] }\end{array}$} \\
\hline & & & & $24 \mathrm{~h}$ & $48 \mathrm{~h}$ & & & & \\
\hline I-36 & + & $\geq 32$ & 0.5 & 8.0 & 32 & 25 & na & $\geq 32$ & na \\
\hline I-33 & + & $\geq 32$ & 0.5 & 8.0 & 16 & 25 & na & $\geq 32$ & na \\
\hline $\mathrm{I}-62$ & + & 0.5 & 0.5 & 2.0 & 2.0 & 1 & na & 0.5 & na \\
\hline $\mathrm{I}-81$ & - & $\geq 32$ & 1.0 & $\geq 256$ & $\geq 256$ & 25 & + & $\geq 32$ & $\geq 32$ \\
\hline I-78 & - & $\geq 32$ & 0.5 & $\geq 256$ & $\geq 256$ & 25 & + & $\geq 32$ & $\geq 32$ \\
\hline $\mid-43$ & - & $\geq 32$ & 0.5 & $\geq 256$ & $\geq 256$ & 25 & + & $\geq 32$ & $\geq 32$ \\
\hline II-61 & - & $\geq 32$ & 0.5 & $\geq 256$ & $\geq 256$ & 25 & + & $\geq 32$ & $\geq 32$ \\
\hline $\mathrm{I}-45$ & - & $\geq 32$ & 0.5 & 32 & 32 & 25 & + & $\geq 32$ & $\geq 32$ \\
\hline $\mathrm{I}-28$ & - & $\geq 32$ & 0.5 & 8 & 32 & 25 & + & $\geq 32$ & 8 \\
\hline $\mathrm{I}-24$ & - & $\geq 32$ & 0.5 & 8 & 16 & 25 & + & $\geq 32$ & $\geq 32$ \\
\hline VIII-44 & - & $\leq 1$ & 0.5 & 2 & 2 & 1 & - & 1 & na \\
\hline $\mid-57$ & - & $\leq 1$ & 0.5 & 2 & 2 & 1 & - & 1 & na \\
\hline IV-74 & - & $\leq 1$ & 0.5 & 2 & 2 & 1 & - & 1 & na \\
\hline VI-3 & - & 0.5 & 0.5 & 2 & 2 & 1 & - & 0.5 & na \\
\hline
\end{tabular}

200 na: not applicable 


\section{References}

1. Arias CA, Murray BE. 2012. The rise of the Enterococcus: beyond vancomycin resistance. Nat. Rev. Microbiol. 10, 266-278

2. Miller WR, Murray BE, Rice LB, Arias, CA. 2016. Vancomycin-Resistant Enterococci: Therapeutic Challenges in the 21st Century. Infect. Dis. Clin. North Am. 30, 415-439

3. Frakking FNJ, Bril WS, Sinnige JC, Klooster JEV, de Jong BAW, van Hannen EJ, Tersmette M. 2018. Recommendations for the successful control of a large outbreak of vancomycin-resistant Enterococcus faecium in a non-endemic hospital setting. J. Hosp. Infect. 100, e216-e225

4. WHO publishes list of bacteria for which new antibiotics are urgently needed. https://www.who.int/news-room/detail/27-02-2017-who-publishes-list-of-bacteria-for-whichnew-antibiotics-are-urgently-needed (last accession date: 10/21/2020)

5. Klare I, Fleige C, Geringer U, Witte W, Werner G. 2012. Performance of three chromogenic VRE screening agars, two Etest(®) vancomycin protocols, and different microdilution methods in detecting vanB genotype Enterococcus faecium with varying vancomycin MICs. Diagn. Microbiol. Infect. Dis. 74, 171-176

6. EUCAST Warning, 10.07.2018, http://www.eucast.org/fileadmin/src/media/PDFs/EUCAST_files/Warnings/Warning_agains t_Low_MIC_V anB_2018.pdf. (last accession date: 04/05/2019)

7. Doernberg SB, Lodise TP, Thaden JT, Munita JM, Cosgrove SE, Arias CA, Boucher HW, Corey GR, Lowy FD, Murray B, Miller LG, Holland TL; Gram-Positive Committee of the Antibacterial Resistance Leadership Group (ARLG). 2017. Gram-Positive Bacterial Infections: Research Priorities, Accomplishments, and Future Directions of the Antibacterial Resistance Leadership Group. Clin Infect Dis. 2017 Mar 15;64(suppl_1):S24S29.

8. Klare I, Bender JK, Fleige C, Kriebel N, Hamprecht A, Gatermann S, Werner G. Comparison of VITEK® 2, three different gradient strip tests and broth microdilution for 
detecting vanB-positive Enterococcus faecium isolates with low vancomycin MICs. 2019. J. Antimicrob. Chemother. 74, 2926-2929

9. Thaker MN, Kalan L, Waglechner N, Eshaghi A, Patel SN, Poutanen S, Willey B, Coburn constitutive resistance during antibiotic therapy. 2015. Antimicrob. Agents Chemother. 59, $1405-1410$

10. Kohler P, Eshaghi A, Kim HC, Plevneshi A, Green K, Willey BM, McGeer A, Patel SN, for the Toronto Invasive Bacterial Diseases Network (TIBDN). 2018. Prevalence of vancomycin-variable Enterococcus faecium (VVE) among vanA-positive sterile site isolates and patient factors associated with VVE bacteremia. PloS One 13, e0193926

11. Sivertsen A, Pedersen T, Larssen KW, Bergh K, Rønning TG, Radtke A, Hegstad K. 2016. A Silenced vanA Gene Cluster on a Transferable Plasmid Caused an Outbreak of Vancomycin-Variable Enterococci. Antimicrob. Agents Chemother. 60, 4119-4127

12. Hill CM, Krause KM, Lewis SR, Blais J, Benton BM, Mammen M, Humphrey PP, Kinana A, Janc JW. 2010. Specificity of Induction of the vanA and vanB Operons in VancomycinResistant Enterococci by Telavancin. Antimicrob. Agents Chemother. 54, 2814-2818

13. Oravcova V, Kolar M, Literak I. 2019. Highly variable vancomycin-resistant enterococci in the north-eastern part of the Czech Republic. Lett. Appl. Microbiol. 69, 16-22

14. Hashimoto Y, Kurushima J, Nomura T, Tanimoto K, Tamai K, Yanagisawa H, Shirabe K, Ike $\mathrm{Y}$, Tomita H. 2018. Dissemination and genetic analysis of the stealthy vanB gene clusters of Enterococcus faecium clinical isolates in Japan. BMC Microbiol. 18, 213

15. Werner G, Klare I, Fleige C, Geringer U, Witte W, Just HM, Ziegler R. 2012. 